\title{
Congenital bronchobiliary fistula
}

INSERM

\section{Source}

INSERM. (1999). Orphanet: an online rare disease and orphan drug data base. Congenital bronchobiliary fistula. ORPHA:2040

Congenital respiratory-biliary fistula (RBF) is a rare developmental defect characterized by an anomalous connection of trachea or bronchus with left hepatic duct presenting with respiratory distress, recurrent respiratory infections and biliary expectoration or vomitus. 